\title{
Power Control for IEEE 802.11 Ad Hoc Networks: Issues and $\mathrm{A}$ New Algorithm
}

\author{
Xiao-Hui Lin, Yu-Kwong Kwok, and Vincent K. N. Lau \\ Department of Electrical and Electronic Engineering \\ The University of Hong Kong, Pokfulam Road, Hong Kong
}

\begin{abstract}
In this paper, we propose an enhancement to the original MAC (multiple access control) protocol in the IEEE 802.11 standard by improving the handshake mechanism and adding one more separate power control channel. With the control channel, the receiver notifies its neighbors about the noise tolerance. Thus, the neighbors can adjust their transmission power levels to avoid packet collision at the receiver. Through extensive simulations on the NS-2 platform, our power control mechanism is found to be effective in that network throughput can be increased by about $10 \%$.

KeyWords: power control, ad hoc networks, IEEE 802.11, medium access control.
\end{abstract}

\section{INTRODUCTION}

In recent years, we have witnessed that the deployment of IEEE 802.11 based ad hoc wireless networks [14], [11] is swiftly proliferating. However, power control mechanism remains a critical challenge in such networks. In the IEEE 802.11/802.11b MAC protocol, there is only one common channel. Each mobile terminal accesses the channel through a CSMA/CA competition mechanism, i.e., a four frame RTS-CTS-DATA-ACK handshake to realize a data transmission. As many other researchers [7] have pointed out, capacity of wireless network is limited by the population density and there is an upper bound in the maximal aggregate channel utilization due to the mutual cochannel interference. Motivated by this observation, in this paper we focus on mechanisms that can increase the network capacity by using an effective power control scheme as shown in Figure 1.

On the problem of transmission power control, numerous researchers have done an extensive volume of work [1], [5], [6], [8], [9], [13], [18], with diverse approaches and motivations. In [16], a power-aware localized routing protocol is proposed to save battery power. In that protocol, the transmission power is controlled based on the distance of the nodes and a power-cost metric is defined. Based on this metric, a minimum energy routing protocol is designed to minimize the total power needed to trans-

This research was supported by a grant from the Hong Kong Research Grants Council under project number HKU 7162/03E.

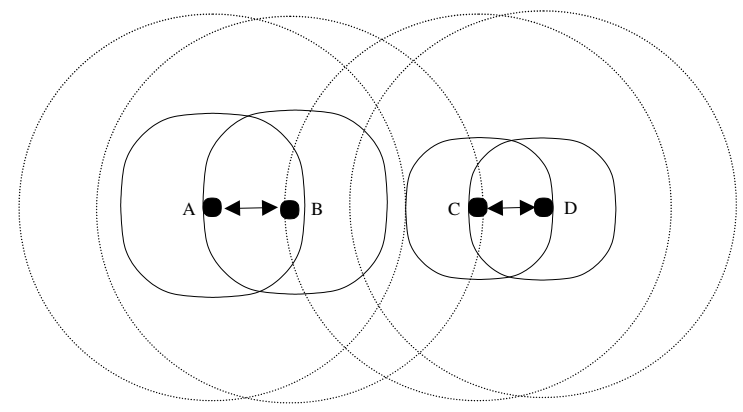

Fig. 1. Judicious power control can allow more simultaneous transmissions with manageable interference.

mit a packet from the source to the destination. Similar work is also reported in [4], mainly focusing on battery power saving. In [5], it is observed that there is a tradeoff relationship between the RF transmission power and the packet retransmission. Reduction in the transmission power leads to the corruption of the packet, thus causing excessive packet retransmissions, which are very power consuming. Thus, it is found that reducing the transmission power cannot necessarily save the battery life. Furthermore, in that paper, an optimal operating point for the system is also reported. In [13], Ramanathan et al. suggest a scheme that can bring about considerable power saving by adjusting the transmission power, thus changing the topology of wireless ad hoc network. Similar work has also been reported in [17], in which, the network topology is dynamically controlled by the transmission power. The new topology resulted from power control increases the network lifetime and reduces the traffic interference by having lower node degrees.

The work by Jung [8] addresses the asymmetrical link problem-different power level by each mobile terminal in a distributed IEEE 802.11 system might cause serious collision, thus deteriorating the network throughput (unfortunately, this phenomenon is also encountered by most of the above mentioned schemes). To tackle this problem, it is suggested that the handshake of RTS-CTS use the normal (maximal) power level, while transmission of the data packet uses the needed power level, during the data transmission, the sender should periodically raise the level to 
the maximal (the period is about $190 \mu \mathrm{s}$ and the duration time of the maximal level is $15 \mu \mathrm{s}$ ). In this manner, the asymmetrical problem is handled satisfactorily and battery power is saved. However, the network throughput cannot be increased.

The above-mentioned research works are mainly targeted at power saving only. In order to improve the channel utilization, Wu and Tseng [18] suggest using dual busy tone multiple access (DBTMA) [3] to realize power control in wireless ad hoc network. In this scheme, each terminal decides the appropriate transmission power level based on the distance between two terminals in a distributed manner. In DBTMA, there are several channels: a control channel, a data channel, and two tones used by the transmitter and receiver to signify their working conditions (receiving or transmitting packet). Upon hearing the busy tones, other mobile terminals can adjust their transmission power to avoid collision, thereby increasing channel utilization. However, the MAC structure of DBTMA is very different from that of IEEE 802.11, and thus, DBTMA is incompatible with most standard devices in the market.

Busy tone scheme is also adopted by PCMA (power controlled multiple access protocol) [9], in which, each mobile terminal is allowed to decide its power level. Different from DBTMA, there is only one data channel (same as IEEE 802.11) and a busy tone. The handshake mechanism is the same as that in IEEE 802.11, i.e., a RTS-CTS signaling exchange precedes the data transmission. When receiving data packet, the receiver periodically sends a busy tone. The loudness of the busy tone is proportional to the noise tolerance at the receiver. On receiving the busy tone, other terminals must compute the noise that it might cause at the receiver when it has a packet to send. Thus, the "hidden terminal" situation [15] is avoided by using this busy tone. However, the busy tone is only used at the receiver to protect the packet corruption by other terminals around it. At the transmitter, transmissions from the surrounding terminals might cause collisions of ACK, thus also leading to retransmissions.

In [1], a minimum table, which records the needed power level to communicate with each neighbor, is kept by every mobile terminal. There is no other auxiliary control channel and busy tone. Each terminal dynamically increases the power level when a transmission fails. This scheme is used in an IEEE 802.11 system, and can enable more TCP connections and save battery power. Indeed, the power control in an IEEE 802.11 environment is challenging in that using different power level at different terminals in a distributed manner introduces asymmetrical link problem [8]. If different power levels are used, packet collision can happen at the receiver (DATA collision) or at the sender (ACK collision). Even a busy tone is adopted in PCMA [9], it can only prevent the data collision at the receiver, but not the ACK collision at the sender. In summary, it is very difficult to design a perfect MAC protocol that can improve both the capacity and power utilization at the same time.

In this paper, we propose a new power control MAC protocol (PCMAC) by adding one more separate power control channel and modifying the handshake mechanism in the original IEEE 802.11. Our goal is to improve network capacity. In our scheme, the sender uses only the needed power level to transmit packet, while the DATA/ACK collision at the receiver/sender side can still be avoided. Through our extensive simulations on NS-2 [10], PCMAC shows improvements in the network capacity. Unlike DBTMA, we only need to do some small modifications on the firmware and software of the IEEE 802.11 protocol, and thus, PCMAC is compatible with the original standard. The rest of this paper is organized as follows. In Section II, we briefly give some background of IEEE 802.11. In Section III, we first discuss the asymmetrical link phenomenon encountered by many previously suggested schemes, and then describe our proposed scheme PCMAC. We present the simulation results in Section IV, together with our interpretations. Section V concludes this paper.

\section{BACKGROUND}

In this paper, we focus on IEEE 802.11 DCF (Distributed Coordination Function), which is a fully distributed medium access control scheme based on CSMA/CA (Carrier Sense Multiple Access with Collision Avoidance). In the scheme, each mobile terminal gets access to the medium on a contention basis. Before a data transmission begins, the sender and receiver must have a RTS-CTS signaling handshake to "reserve" the channel. The whole transmission sequence is a RTS-CTS-DATAACK four-way handshake as illustrated in Figure 2.

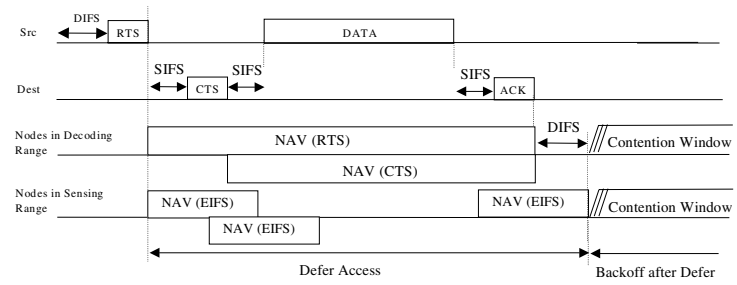

Fig. 2. Illustration of the handshake mechanism in a standard IEEE 802.11 environment.

When a sender has a packet to transmit, it senses the channel by detecting the air interface (in the physical layer) and looking up its NAV (Network Allocation Vec- 
tor). If the channel is busy, the terminal waits until the channel becomes free, in which case it sends a RTS to the destination terminal. On successfully receiving the RTS, the destination replies the source with a CTS. The source can begin data transmission after the CTS is received. After the data is received at the destination, the destination sends an ACK to the source, confirming the success of a data reception. This is an ideal case of a four-way handshake. If the source fails to receive CTS or ACK (collision at source or destination), it backs off for a random period of time by doubly increasing its contention window $(\mathrm{CW})$ size.

Each packet, including RTS, CTS, DATA, and ACK, has a duration time in its header, which is used to specify the time that the wireless channel will still be occupied. The terminals in the neighborhood, on receiving these packets, adjust their NAVs as illustrated in Figure 2. Thus, the wireless channel is deemed being occupied by a terminal if either its physical air interface or the NAV indicates so. Based on the characteristics of wireless propagation model, the data reception area can be further divided into two zones due to the signal attenuation [8]: decoding zone and carrier sensing zone, as shown in Figure 3. Decoding zone is the area, within which, the receiver can receive and correctly decode the packet. Within this zone, the received signal strength is greater than $R x_{T h}$, which is the minimum power level required to correctly decode the received packet. On the other hand, the carrier sensing zone is the area, within which, the received power level is less than $R x_{T h}$, but greater than $C S_{T h}$, which is the required minimum power level to sense the received packet (power level below $C S_{T h}$ is treated as noise). Thus, within carrier sensing zone, the received packet can only be "felt," but not decoded.

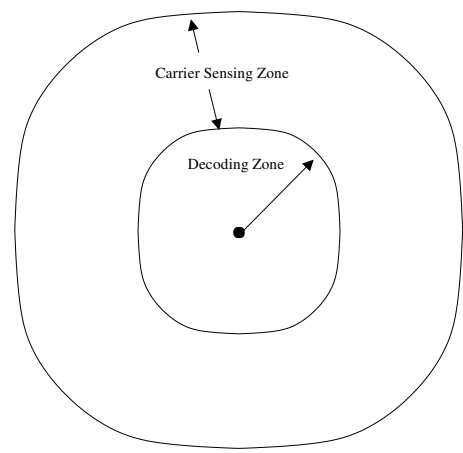

Fig. 3. Decoding zone and carrier sensing zone centered at the sender.

In Figure 2, it can be seen that each mobile terminal within decoding zone adjusts its NAV to avoid collision at source or destination based on the received packet (e.g., RTS, CTS). But terminals in carrier sensing zone can only "feel" these packets, and cannot correctly decode them. Thus, such terminals may still cause collision of the ACK at the source or DATA collision at the destination. To address this problem, IEEE 802.11 specifies that, the terminals in the carrier sensing zone continuously back off for EIFS period by adjusting their NAVs whenever they can sense the carrier but cannot decode it. That means, when a terminal can sense a transmission but cannot decode it, must set its NAV for EIFS duration [8]. Note that EIFS duration is longer than the transmission time of an ACK. The purpose of doing this is to protect the ACK collision at the source. As the parameters used in NS-2 [10] and reference [8], the ranges for decoding and carrier sensing zone are $250 \mathrm{~m}$ and $550 \mathrm{~m}$, respectively, when using the normal (maximal) power level. It should be noted that these two ranges are dynamically changed when using different transmission power levels. This can lead to an asymmetrical link problem: some terminals beyond the carrier sensing zone cannot adjust their NAVs because the sender is transmitting packet with a relatively lower power level, thus causing packet collisions [8] as illustrated in Figure 4. This phenomenon and solution to this problem are further elaborated below.

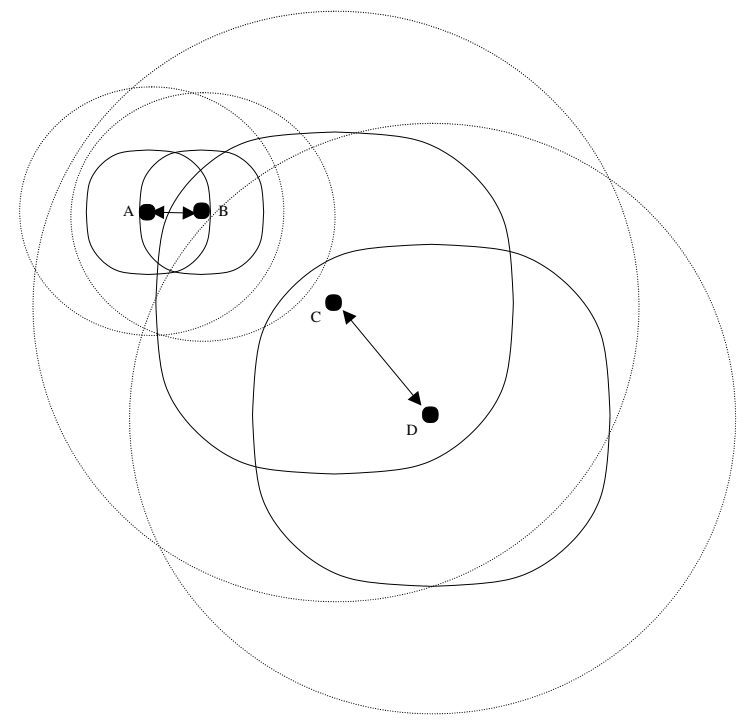

Fig. 4. Asymmetrical link problem: terminals $\mathrm{C}$ and $\mathrm{D}$ are outside the carrier sensing zone of $\mathrm{A}$ and $\mathrm{B}$; and thus, $\mathrm{C}$ cannot sense the signals sent by A or B. C can cause packet collision problems to B if C's transmission power is high enough.

\section{ASYMMETRICAL LINK PHENOMENON AND PCMAC PROTOCOL}

Different transmission power levels by different mobile terminals in a fully distributed manner introduce asymmetrical link phenomenon. This is because compared with the 
original fixed normal (maximal) power level, using different power levels causes the decrease of the decoding and carrier sensing area. When the neighboring terminals cannot decode or sense the packet (because they are outside the decoding and sensing zone), they cannot adjust their NAVs, thus they mistakenly consider that the wireless channel is free and transmit their own packets, leading to collisions. This scenario is depicted in Figure 4. We can see that there are two source and destination pairs: $\mathrm{A} \leftarrow \mathrm{B}$ and $\mathrm{C} \leftarrow \mathrm{D}$. Terminals $\mathrm{C}$ and $\mathrm{D}$ are outside the decoding and sensing zones of $\mathrm{A}$ and $\mathrm{B}$, so they cannot even sense the transmitted signals between $\mathrm{A}$ and $\mathrm{B}$. When there is data transmission between $\mathrm{A}$ and $\mathrm{B}$, such a transmission is not sensed by $\mathrm{C}$ and $\mathrm{D}$, and thus, the transmitted data between A and B get corrupted by the terminal C's transmission.

Some researchers also mention another "basic" power control scheme to save battery power [8], in which, the RTS-CTS dialogue uses the normal (maximal) power level, while DATA-ACK uses the minimal needed power level. This scheme is shown in Figure 5. In this manner, the wireless channel is first "reserved" by RTS-CTS, and the potential terminals in the maximal decoding zone of the sender and receiver can adjust their NAVs when receiving RTS or CTS so that the probability of packet collision is greatly decreased. However, the drop of DATA transmission power level also results in the shrink of sensing zone. When the terminals in the original sensing zone cannot sense the signal, they might think that channel is free and transmit their packets, thus causing the packet collision. This is also an example of asymmetrical link phenomenon. The scenario is depicted in Figure 6. The same observation and analysis can also be found in [8].

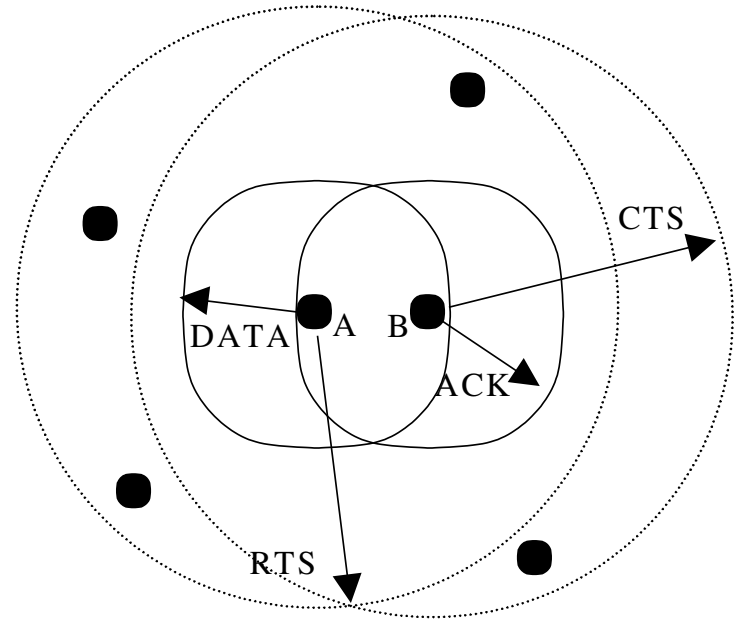

Fig. 5. RTS and CTS are transmitted at the normal power level; while DATA and ACK are transmitted at the required power level.

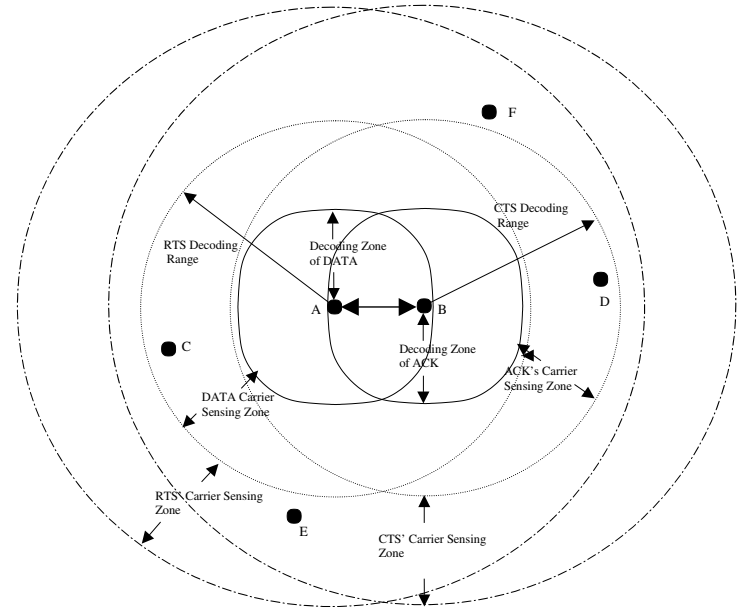

Fig. 6. Terminal A sends data packets to B. RTS and CTS are transmitted at the normal power level; while DATA and ACK are transmitted at the required power level. The reduction of power level for DATA and ACK causes the shrinking of the carrier sensing zone. Thus, terminal $\mathrm{E}$ and $\mathrm{F}$ cannot sense the DATA and ACK, and hence, collisions can occur if $\mathrm{E}$ and $\mathrm{F}$ transmit at a high enough power.

Asymmetrical link phenomenon causes the ineffective usage of the wireless channel resource, thus leading to serious consequences: (1) the frequent data collisions, resulting in more retransmissions, which is a waste of the limited wireless bandwidth and battery power; (2) deterioration in network performance, in that capacity is decreased and packet delay is significantly increased; (3) unfairness in the wireless channel usage, e.g., in Figure 4, the transmission between A and B is frequently suppressed by $\mathrm{C}$ and $\mathrm{D}$, between which, a much higher power level is needed.

The challenging points of power control in an IEEE 802.11 system are: (1) eliminating the collision at both sides (DATA collision at receiver side and ACK collision at sender side), under the asymmetrical links environment; (2) eliminating the collision without the sacrifice of the network capacity; (3) ensuring the fairness among all sender-receiver pairs, i.e., the communication pair using higher power level should not suppress the nearby communication pair using relatively lower power level. An excellent power control scheme should satisfy all these goals simultaneously. However, most of the schemes cannot satisfy all of them. In this regard, we propose a new power control medium access control protocol-PCMAC, which greatly eliminates the negative effects incurred from the asymmetrical links. In the proposed PCMAC scheme, RTS, CTS, DATA, and ACK, are transmitted at the needed power level.

In cellular networks, to ensure that each mobile terminal has the same receive power level at the base station, 
the base station continually receives and estimates the signal strength from a particular mobile user. Based on the signal noise ratio of a particular mobile user at base station, the base station instructs the mobile terminal to adjust its transmission power, through a forward control subchannel [15]. For example, in IS-95, for every $1.25 \mathrm{~ms}$ the base station instructs a mobile terminal having a power level adjustment, and the adjustment step is $1 \mathrm{~dB}$. This is a centralized power control scheme using a forward control channel. Inspired by this scheme, in PCMAC, we add a separate power control channel, with bandwidth of 500 kbps, into the IEEE 802.11 system. When a mobile terminal begins to receive data packet, i.e., after a RTS-CTS exchange, it estimates the signal and noise strength, computing the noise level it can still endure by $\frac{P_{r}}{S I R_{t h}}-P_{n}$, and then broadcasts this information through the power control channel at the normal (maximal) level to its surrounding terminals. Here, $P_{r}$ and $P_{n}$ are the received signal power and noise power observed at the receiver side, and $S I R_{T h}$ is capture threshold (i.e., the required signal to noise ratio above which the receiver can achieve successful decoding). With this information, a nearby terminal calculates whether its transmission might cause corruption at this particular terminal that is currently receiving data packet. If yes, it must back off until the current reception is completed; otherwise, it can begin its own transmission. In this manner, the probability of data collision is greatly reduced at the receiver.

This power control channel is only used at the receiver to protect data packet from collision. However, at the sender, there might be many potential mobile terminals beyond the sender's sensing zone, and they might cause ACK corruption. Now here comes the question: how to avoid the ACK collision at the sender side? Our solution is: modifying the original RTS-CTS-DATA-ACK four-way handshake to a three-way handshake, i.e., a RTSCTS-DATA dialogue. Thus, the ACK collision problem at the sender is completely eliminated. However, without the ACK, we need to come up with another mechanism to handle the acknowledgment aspect. Our solution is to let every terminal keep two tables: sent-table and received-table, storing information about the packets sent and received, respectively. When a sender transmits data packet to a particular receiver, it should record the session ID (session ID stands for the particular source destination pair) and sequence number of this packet, together with the ID of the particular receiver, in its sent-table. The sender also keeps a copy of this packet for the future retransmission (if there is such a need). The same, when receives a data packet, the receiver records the session ID and sequence number of the received packet, together with the ID of the sender, from whom, it receives this packet, in its received-table. Thus, when a receiver $B$ receives a RTS form a particular sender A, it replies sender B with a CTS, which includes the session ID together with the sequence number of the last received data packet from $\mathrm{A}$. When $\mathrm{A}$ receives this CTS, it checks these two fields, comparing them with those recorded in its sent-table. If the two match, this means that the last data packet has been already successfully received by B; otherwise, A retransmits the last sent data packet to $\mathrm{B}$ (note that every time a data packet is transmitted, it has a copy at the sender). However, this three-way handshake mechanism only applies to data packet. For the unicast of routing packet, we still use four-way handshake mechanism, i.e., a RTS-CTS-DATA-ACK process.

The route entry at each terminal might be updated from time to time due to the network mobility, thereby the upstream or downstream terminal for a particular terminal is also changed: a route entry is set up with the reception of a RREP (route reply packet used by network layer), or deleted with the reception of a RRER (route error packet used by network layer) [12]. The reception of a RREP or RRER means the new beginning or the break of a particular session at this terminal, respectively. Thus, the sentand received-tables are maintained in the following manner: every time a terminal successfully sends a RREP to a downstream terminal, its received-table as to this downstream terminal is reset (session number and last received data packet sequence number are set to null). On the other hand, when a terminal receives a RRER from an upstream terminal, its received-table as to this upstream terminal is also reset (session number and last sent data packet sequence number are set to null), and the copy of last sent data packet to this upstream terminal is deleted.

In PCMAC, we transmit RTS-CTS-DATA-(ACK) at the minimal needed power level, while a broadcasting packet at the normal (maximal) power level. In the packet head of a RTS, CTS, and unicast/broadcasting packet, the power level at which this packet is transmitted is included. Each mobile terminal also keeps a power history table, recording the needed power level to reach every other terminal. Once receiving a packet from some sender, the terminal detects the signal strength $E$ of this packet, computing the needed power level if it has packet to this sender (note that received packet include the power level at which it is transmitted) as: $P_{n e e d}=\frac{R x_{t h} P_{T}}{E}$, where $E$ is the observed signal strength at the receiver, and $R x_{T h}$ is the minimal power threshold that can satisfy correct decoding, while $P_{T}$ is the power level at which the packet is transmitted. The table can be very small because it is unlikely that the terminal concurrently communicates with many others terminals at the same time. With respect to each terminal in the table, 
the record has an expiration time ( 3 seconds), i.e., if the record has not been updated within the expiration time, it is deleted. If a terminal A wants to communicate with another terminal B, and A has no power level record as to B in its table, A uses the normal power level to transmit the packet.

To summarize, due to the introduction of asymmetrical links, the core issue we need to tackle in power control is: how to protect the DATA collision at the receiver side and ACK collision at the sender side, after the RTS-CTS exchange? The proposed PCMAC tackles this problem in this manner: using the power control channel to prevent DATA collision at the receiver side, and modifying the handshake mechanism to eliminate ACK collision at the sender side (ACK is not used and ACK collision does not exist). Before giving the detailed elaboration of PCMAC, some assumptions are made:

1. The power control channel has no interference with the data channel. The two channels share the same propagation characteristics, i.e., have the same attenuation and fading parameters, and the transmission ranges are same if using the same power level.

2. The propagation conditions (attenuation, fading, etc.) between source and destination terminal is assumed to be the same in both directions, or the propagation gain in both directions are the same $G_{i j}=G_{j i}$.

3. There is also collision in the power control channel. To decrease the collision probability, the length of broadcast packet should be kept short. Thus, the packet only includes the terminal ID and the noise endurance at the receiver. The packet frame structure is shown in Figure 7.

\begin{tabular}{|c|c|c|c|}
\multicolumn{1}{c|}{16 bits } & \multicolumn{1}{c}{8 bits } & 16 bits & 8 bits \\
\hline Preamble & Node ID & Noise Tolerance & FEC \\
\hline
\end{tabular}

Fig. 7. Frame structure of the power control packet.

4. The length of the data packet is fixed (512 bytes in our experiment), such that upon receiving power control signaling from the control channel, other terminals know the left duration time of this data reception.

5. The transmission power is limited by the most vulnerable neighbor (with the smallest signal noise ratio).

The following is a step-by-step description of PCMAC.

Step 1: When a mobile terminal A has packet to send to terminal $\mathrm{B}$, it checks whether the wireless channel is now busy by detecting the physical air interface and the NAV. If the channel is temporarily being used, it backs off and keeps monitoring the channel until it is freed. If the channel is free, it further looks up its power history table, to see with which power level it should use to get to the terminal B. Assume that the power level is $P_{A B}$.

Step 2: Terminal A computes whether using power level might cause collision at the nearby receivers. Or such a constraint must be satisfied: for each nearby current receiver known by $\mathrm{A}$, say $\mathrm{C}$, the caused noise level at $\mathrm{C}$ $G_{A C} P_{A B} \leq 0.7\left(\frac{P_{r c}}{S I R_{T h}}-P_{n c}\right)$. Here, $G_{A C}$ is the propagation gain from $\mathrm{A}$ to $\mathrm{C}$, and $G_{A C} P_{A B}$ is the caused noise by $\mathrm{A}$ at $\mathrm{C}, \frac{P_{r c}}{S I R_{T h}}-P_{n c}$ is the noise tolerance at $\mathrm{C}$ and is known by A. We choose coefficient of 0.7 because: (1) the noise level might be fluctuating at the $\mathrm{C}$ (although through our observation, this fluctuating scope is rather small in short span of a data reception, about $2.2 \mathrm{~ms}$ ); and (2) there might be other terminals also wanting to transmit at the same time. Thus, we should leave some redundancy in the noise tolerance at terminal C. If this constraint cannot be satisfied, terminal A must back off until the reception is completed. Otherwise, terminal A can send RTS out, waiting for CTS from C. This RTS also includes the noise level $P_{n A}$ at terminal A and the power level $P_{T A}$ at which RTS is transmitted. If timeout and A cannot receive CTS from B, A increases its power level (by one class until gets to maximal level) and repeat the computation as mentioned above.

Step 3: If terminal B receives the RTS, it should reply with CTS, which should be transmitted at the power level of $\max \left\{\frac{R x_{T h} P_{T A}}{E_{B A}}, \frac{S I R_{T h} P_{n A}}{G_{A B}}\right\}$, so that this CTS can be captured and received at source A. Here, $E_{B A}$ is the observed received RTS power at $\mathrm{B}$, and $G_{A B}$ is the propagation gain which can be computed based on $P_{T A}$ and $E_{B A}$. In order that the following DATA from terminal A can be also captured and received at B, B required DATA be sent at the power level $\max \left\{\frac{R x_{T h} P_{T A}}{E_{B} A}, \frac{S I R_{T h} P_{n B}}{G_{A B}}\right\}$. B also put this information into the CTS. Before transmitting CTS, terminal B must also do the collision computation same as terminal A, so as to avoid collision at the surrounding receivers. If B is allowed to send CTS, it appends to CTS the session ID, together with the sequence number of the last data packet received from A, then sends this CTS to A.

Step 4: When terminal A receives CTS, it compares the session ID and sequence number included in CTS with those stored in its sent-table, to have a successful reception check of the last sent packet. If both match, terminal A transmits the next data packet to $\mathrm{B}$, and updates its senttable by storing the related information of this next data packet in the sent-table. If these two fields do not match, terminal A has to retransmit the last sent data packet to B. Before transmitting the DATA packet at the required power level, terminal A again repeats the collision computation.

Step 5: When terminal B begins to receive data packet, it estimates the signal and noise strength, computing the noise level it can still endure by $\frac{P_{r_{B}}}{S I R_{T h}}-P_{n B}$, and then 
broadcast this information out through the power control channel at the normal power level.

Step 6: If terminal B successfully receives this data packet, it updates its received-table by storing the session ID and sequence number in it.

Step 7: Terminal B can choose to reply A with an ACK, if the received packet is not a data packet (e.g., is a RREP or RRER), or just return to IDLE state, if the received packet is DATA.

The transmission of other unicast packets (non-data packet, such as RREP or RRER) is similar to that of a data packet, except that there is no need to have a check of last sequence number and session ID, and the receiver has to reply the sender with an $\mathrm{ACK}$ to confirm the successful reception.

\section{Simulation ENVIRONMENT ANd Results}

In order to test the performance of PCMAC, we use NS2 (Version ns2.1b8a), a discrete event simulator extended by CMU Monarch project to support ad-hoc routing, as our simulation platform. NS-2 contains a complete set of ad hoc routing protocols and can support IEEE 802.11 MAC standard that executes a wireless RF physical layer operating at a $914 \mathrm{Mhz}$, with a data rate of $2 \mathrm{Mbps}$. All the wireless physical layer parameters in the simulator have been tuned to model the Lucent Wavelan card. In NS-2 the decoding and sensing ranges are $250 \mathrm{~m}$ and $550 \mathrm{~m}$, respectively, when using the normal power level.

We choose the basic IEEE 802.11 without power control and two schemes with power control as our references. In Scheme 1, RTS and CTS are transmitted at the normal power level, while DATA and ACK are transmitted at the needed power level. In Scheme 2, all the packets, including RTS, CTS, DATA and ACK are transmitted at the needed power level. The broadcast packets are transmitted at the normal power level in all protocols, including Scheme 1 and Scheme 2, PCMAC and basic 801.11. In Scheme 1 and Scheme 2, each mobile terminal also keeps a power history table as in PCMAC, and the table update mechanism is also similar to that of PCMAC. We choose Scheme 1 and Scheme 2 as our references because they are adopted by many other power control algorithms [1], [2], [4], [5], [16], [17], in which, there exists asymmetrical link problem.

Same as the parameters used in reference [8], in our simulation, we adopt ten transmission power levels: $1 \mathrm{~mW}, 2$ $\mathrm{mW}, 3.45 \mathrm{~mW}, 4.8 \mathrm{~mW}, 7.25 \mathrm{~mW}, 10.6 \mathrm{~mW}, 15 \mathrm{~mW}, 36.6$ $\mathrm{mW}, 75.8 \mathrm{~mW}$, and $281.8 \mathrm{~mW}$, which roughly correspond to the decoding range of $40 \mathrm{~m}, 60 \mathrm{~m}, 80 \mathrm{~m}, 90 \mathrm{~m}, 100$ $\mathrm{m}, 110 \mathrm{~m}, 120 \mathrm{~m}, 150 \mathrm{~m}, 180 \mathrm{~m}$, and $250 \mathrm{~m}$, respectively, when the two-way ground propagation model (see ns man- ual [10]) is adopted. The simulation parameters we used are as follows:

- number of terminal: 50 ;

- testing field: $1000 \mathrm{~m} \times 1000 \mathrm{~m}$;

- mobile speed: $3 \mathrm{~m} / \mathrm{s}$;

- mobility model: random way point, i.e., when the terminal reaches its destination, it pauses for 3 seconds, then randomly chooses another destination point;

- bandwidth of the power control channel: $500 \mathrm{kbps}$;

- traffic model: continuous bit rate (CBR), using UDP with packet size of 512 Bytes, and 10 source and destination pairs in the network.

- simulation time: 400 seconds; and

- routing protocol: AODV [11], [12], which has been implemented into NS-2.

To evaluate the four MAC protocols, we increase the traffic load until the network get saturated, comparing them by using the following metrics:

- Aggregate Network Throughput: average number of data packets arrives at their destinations per second in the whole network scale, measured in kbps.

- Average End-to-End Delay: Measured in ms, the end-toend delay stands for the duration time for a packet transmitted from its source to the destination.

We test all the MAC protocols under a relatively low mobility environment, because our focus is put on seeing how MAC protocols can influence the above mentioned metrics, not how routing protocol reacts in a high mobility environment. High mobility might "blur" our sights and more network overhead is generated.

Figure 8 shows the increase of aggregate network throughput with the increase of traffic load. We can see that with an appropriate power control scheme, PCMAC has the highest network throughput among four MAC protocols. By using PCMAC, the network capacity has an improvement of about 8-10\%, compared with that of basic IEEE 802.11, which is an unmodified MAC protocol without power control. Adopting power control can realize wireless channel spatial reuse, thus allowing more simultaneous transmissions. This, of course, increases the network capacity. However, in the power control, packet collision due to asymmetrical link problem must be properly tackled. In Scheme 1, the transmission of RTS-CTS is with the normal power level, but the drop of power level with DATA-ACK causes the shrink of sensing zone. Thus terminals outside the sensing zone might cause collision at both sides, as illustrated in Figure 6. In Scheme 2, however, all non-broadcast packets are transmitted at the needed power. This introduces more asymmetrical links, thus more packet collisions happen than in Scheme 1. Collision incurs the retransmission of the packet, which is a 
waste in the limited wireless bandwidth, thus decreasing the network capacity.

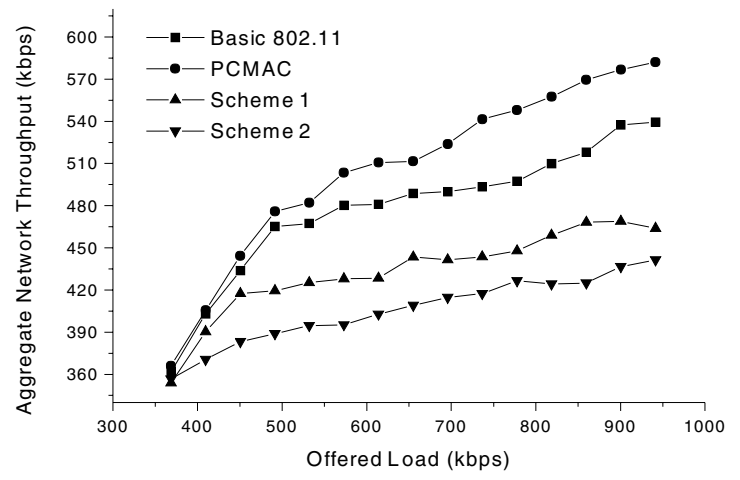

Fig. 8. Aggregate network throughput versus offered load.

Figure 9 illustrates the average packet end to end delay versus the increased traffic load. In all protocols, the end to end delay increases with the load because the network gets more congested. Due to the judicious power control in PCMAC, packet delay in PCMAC is the shortest. With an appropriate power control scheme, wireless resource management is more reasonable, and channel spatial reuse decreases the packet queuing time (waiting for the availability of the channel) in its buffer, thus shortening the end to end delay. However, in Scheme 1 and Scheme 2, frequent packet collision incurs the retransmission of the packet, which increases the packet delay. We can also see that the asymmetrical link problem seems more serious in Scheme 2 than in Scheme 1.

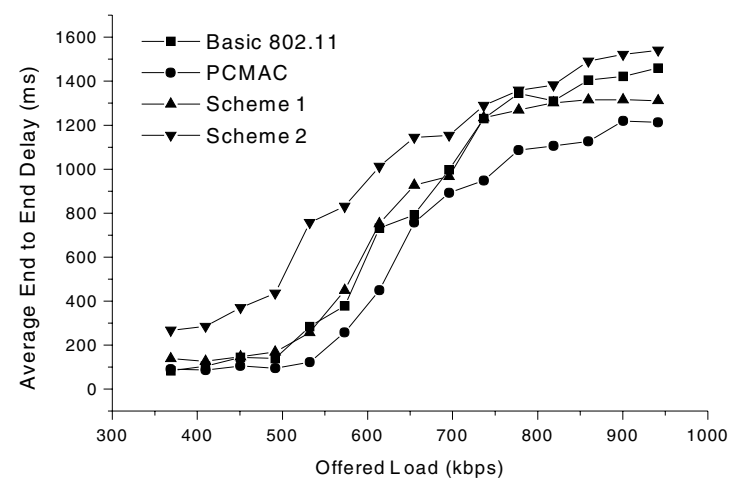

Fig. 9. Average end-to-end delay versus offered load.

\section{Conclusions}

In this paper, we have presented our power control MAC protocol called PCMAC, which can effectively tackle the asymmetrical link problem commonly encountered by other power control schemes. Through extensive simulations, PCMAC has illustrated its superiorities in that network capacity is increased. Furthermore, without great modification in the firmware and software, PCMAC can be incorporated in the existing IEEE 802.11 standard, making it practicable in a real environment.

\section{REFERENCES}

[1] S. Agarwal, S. V. Krishnamurthy, R. H. Katz, and S. K. Dao, "Distributed Power Control in Ad Hoc Wireless Networks," Proc. 2001 IEEE Int'l Symposium on Personal, Indoor and Mobile Radio Communications (PIMRC'2001), pp. 59-66, Sept. 2001.

[2] P. Chen, B. O'Dea, and E. Callaway, "Energy Efficient System Design with Optimum Transmission Range for Wireless Ad Hoc Networks," Proc. ICC'2002, vol. 2, pp. 945-952, Mar. 2002.

[3] J. Deng and Z. J. Hass, "Dual Busy Tone Multiple Access (DBTMA): A New Medium Access Control for Packet Radio Networks," Proc. Int'l Conference of Universal Personal Communications Oct. 1998.

[4] S. Doshi, S. Bhandare, and T. X. Brown, "An On-Demand Minimum Energy Routing Protocol for a Wireless Ad Hoc Network," Mobile Computing and Communication Review, vol. 6, no. 3, pp. 50-66, Mar. 2002.

[5] J. P. Ebert and A. Wolisz, "Combined Tuning of RF Power and Medium Access Control for WLANs," Proc. 1999 IEEE Int'l Workshop on Mobile Multimedia Communications (MoMuC'99), pp. 74-82, Nov. 1999.

[6] T. A. Elbatt, S. V. Krishnamurthy, D. Connor, and S. Dao, "Power Management for Throughput Enhancement in Wireless Ad-hoc Networks," Proc. ICC'2000, vol. 3, pp. 1506-1531, June 2000.

[7] P. Gupta and P. R. Kumar, "The Capacity of Wireless Networks," IEEE Trans. Information Theory, vol. 46, no. 2, pp. 388-404, Mar. 2000.

[8] E.-S. Jung and N. H. Vaidya, "A Power Control MAC Protocol for Ad Hoc Networks," Proc. ACM 8th Annual International Conference on Mobile Computing and Networking (MOBICOM'2002), pp. 36-47, Sept. 2002.

[9] J. P. Monks, V. Bharghavan, W. Mei, and W. Hwu, "A Power Controlled Multiple Access Protocol for Wireless Packet Networks," Proc. INFOCOM'2001, pp. 219-228, Apr. 2001.

[10] The Network Simulator (NS-2), http://ww.isi.edu/nsnam/ns/, 2002.

[11] C. E. Perkins, Ad Hoc Networking, Addison-Wesley, 2001.

[12] C. E. Perkins and E. M. Royer, "Ad-hoc On-Demand Distance Vector Routing, Mobile Computing Systems and Applications," Proc. WMCSA'99, pp. 90-100, 1999.

[13] R. Ramanathan and R. Rosales-Hain, "Topology Control of Multihop Wireless Networks using Transmit Power Adjustment," Proc. INFOCOM'2001, pp. 404-413, Apr. 2001.

[14] E. Royer and C. K. Toh, "A Review of Current Routing Protocols for Ad Hoc Mobile Wireless Networks," IEEE Personal Communications, vol. 6, no. 2, pp. 46-55, Apr. 1999.

[15] T. S. Rappaport, Wireless Communications: Principles and Practice, Prentice-Hall, 1996.

[16] I. Stojmenovic and X. Lin, "Power-Aware Localized Routing in Wireless Networks," IEEE Trans. Parallel and Distributed Systems, vol. 12, no. 11, pp. 1122-1133, Nov. 2001.

[17] R. Wattenhofer, L. Li, P. Bahl, and Y.-M. Wang, "Distributed Topology Control for Power Efficient Operation in Multihop Wireless Ad Hoc Networks," Proc. INFOCOM'2001, pp. 404413, Apr. 2001.

[18] S.-L. Wu, Y.-C. Tseng, and J.-P. Sheu, "Intelligent Medium Access for Mobile Ad Hoc Networks with Busy Tones and Power Control," IEEE Journal on Selected Areas in Communications, vol. 18, no. 9, pp. 1647-1657, Sept. 2000. 